\title{
Fuzzy Kalman Filter Validation Using the Local Statistical Approach
}

\author{
G. Rigatos ${ }^{1} \cdot$ P. Siano ${ }^{2}$
}

Received: 7 January 2015 / Revised: 24 March 2015 / Accepted: 18 April 2015 / Published online: 24 May 2015

(C) Springer Science+Business Media Singapore 2015

\begin{abstract}
In fuzzy Kalman filtering, each local Kalman filter makes use of its own model about the system's dynamics and the local state estimates are fused into one single state estimate through fuzzy weighting. These models can be inaccurate or the system's dynamics may change. The objective of the paper is to provide a method for finding out which one of the models used by the local Kalman filters contains parameters that deviate from the nominal values of the parameters of the real system. This is a problem of validation of the fuzzy Kalman filter, that is solved with the use of the local statistical approach to fault diagnosis. The development of a tool which can detect incipient changes in the parameters of the fuzzy Kalman filter is important, since such types of distributed Kalman filters are used in numerous applications that need precision and reliability in the provided state estimation (sensor networks, navigation systems, industrial production systems etc.).
\end{abstract}

Keywords Model validation - Nonlinear state estimation · Nonlinear Kalman filtering · Fuzzy local linearization . Local statistical approach to fault diagnosis

\footnotetext{
G. Rigatos

grigat@ieee.org

P. Siano

psiano@unisa.it

1 Unit of Industrial Automation, Industrial Systems Institute, 26504 Rion Patras, Greece

2 Department of Industrial Engineering, University of Salerno, 84084 Fisciano, Italy
}

\section{Introduction}

Fuzzy Kalman filtering is a method for distributed state estimation in which the local state estimates produced by distributed Kalman filters are fused into one single state estimate through weighting with fuzzy membership values. The fuzzy Kalman filter is designed based on the coverage of the state space by several local linear estimators according to the concept of fuzzy local linearization. It can be shown that the fuzzy Kalman filter can be written in the form of a Takagi-Sugeno-Kung (TSK) fuzzy model, which stands for local ARMAX models weighted by fuzzy membership functions [1-4]. The development of a method for validation of the fuzzy Kalman filter and for detecting incipient changes in its parameters, is important for a large number of applications in which such a type of distributed filtering is used (environmental surveillance, autonomous navigation systems, decentralized monitoring of industrial production etc.).

The problem of Kalman filter's validation has been studied also in past, however the developed methods are limited to linear systems and give no evidence that incipient parameters change is possible. Indicative results on validation and consistency checking of Kalman filters can be found in [511]. Actually in [5-7], the $\chi^{2}$ test and other statistical indexes are used for testing the accuracy of the estimation provided by the linear Kalman filter, whereas in [8-10] these methods are applied to multiple and distributed Kalman filters and to the Ensemble Kalman filter. In [11] statistical methods are developed, in the form of a residuals and a smoother test, to check inconsistencies in the Kalman filter. Other frequently met tests used in the assessment of Kalman filter's accuracy and consistency are (i) the normalized error square (NES) test, (ii) the autocorrelation test and (iii) the normalized mean error test [12-14]. If the aforementioned tests are 
not satisfied then this means that the Kalman filter is not running optimally, and the filter has to be retuned, or that the filter's design has to be reconsidered.

In this paper the local statistical approach to fault diagnosis is proposed as a method for validation of fuzzy Kalman filtering. This method, first appearing in [15], has been successfully tested in a wide range of fault diagnosis problems as shown in [16-18], while some of its recent applications in nonlinear and distributed parameter systems can be found in $[19,20]$. Here, the local statistical approach is used to check whether the models of the local Kalman filters that constitute the fuzzy Kalman filter remain consistent with respect to parameters of the real system. The paper provides one of the few approaches for testing the accuracy of distributed Kalman filters and the only one that permits to detect parametric changes that are of magnitude of less than $1 \%$ of the nominal value of the parameter.

Comparing to the previously mentioned methods for Kalman filter validation, the proposed approach is a noteworthy contribution to this area. Validation of the fuzzy Kalman filter with the Local Statistical Approach has two significant advantages: (i) it provides a credible criterion (upgraded $\chi^{2}$ test) to detect if the fuzzy Kalman filter is acceptable or not, no matter what the distribution of the measurement data is. This criterion is more efficient than the aforementioned normalized square error and mean error tests since it employs the modeling error derivative and records the tendency for change. Thus early change detection for faults in the filter's parameters becomes possible (ii) it recognizes the parameters of the fuzzy Kalman filter that are responsible for the deviation of the filter's estimates from the real output of the monitored dynamical system. Thus the retuning or redesign of the fuzzy Kalman filter can focus on a small number of its parameters.

The structure of the paper is as follows: In "Consistency of the Kalman Filter" section previous methods of consistency checking and accuracy assessment of the Kalman filter are provided. The results are focused on the linear Kalman filter algorithm. In "Equivalence Between Kalman Filters and Regressor Models" section the equivalence of Kalman filtering to linear regressors of the ARMAX type are given. It is also shown that fuzzy Kalman filters are equivalent to a weighted sum of linear ARMAX regressors which finally take the form of a TSK neurofuzzy model. In "Change Detection with the Local Statistical Approach" section the local statistical approach to fault diagnosis is proposed as a statistical method that enables to perform consistency checking of the fuzzy Kalman filter. In "Simulation Tests" section simulation tests are provided about validation of the fuzzy Kalman filter, showing the capability of the proposed statistical method to detect and isolate the local model in the fuzzy Kalman filter that contains faulty parameters. Finally in "Conclusion" section concluding remarks are stated.

\section{Consistency of the Kalman Filter}

\subsection{The Discrete-Time Kalman Filter for Linear Dynamical Systems}

In the discrete-time case a dynamical system is assumed to be expressed in the form of a discrete-time state model [21-23]:

$$
\begin{aligned}
x(k+1) & =A(k) x(k)+B(k) u(k)+w(k) \\
z(k) & =C x(k)+v(k)
\end{aligned}
$$

where the state $x(k)$ is a $m$-vector, $w(k)$ is a $m$-element process noise vector and $A$ is a $m \times m$ real matrix. Moreover the output measurement $z(k)$ is a $p$-vector, $C$ is an $p \times m$ matrix of real numbers, and $v(k)$ is the measurement noise. It is assumed that the process noise $w(k)$ and the measurement noise $v(k)$ are uncorrelated.

Now the problem of interest is to estimate the state $x(k)$ based on the sequence of output measurements $z(1), z(2)$, $\ldots, z(k)$. The initial value of the state vector $x(0)$, and the initial value of the error covariance matrix $P(0)$ are unknown and estimations of them are considered, i.e. $\hat{x}(0)=$ a guess of $E[x(0)]$ and $\hat{P}(0)=$ a guess of $\operatorname{Cov}[x(0)]$.

For the initialization of matrix $P$ one can set $\hat{P}(0)=\lambda I$, with $\lambda>0$. The state vector $x(k)$ has to be estimated taking into account $\hat{x}(0), \hat{P}(0)$ and the output measurements $Z=$ $[z(1), z(2), \ldots, z(k)]^{T}$, i.e. $\left.\hat{x}(k)=\alpha_{n}(\hat{x}(0)), \hat{P}(0), Z(k)\right)$. This is a linear minimum mean squares estimation problem (LMMSE) formulated as $\hat{x}(k+1)=a_{n+1}(\hat{x}(k), z(k+1))$ (variable $\alpha_{n}$ actually denotes that in Kalman filter-based estimation the estimate of the state vector obtained at time instant $k+1$ is a function of the initial value of the state vector estimate and of the estimation error covariance matrix. It also depends on the sequence of output measurements $Z$ that has provided the state estimate up to the $k$-th time instant, which means that the estimation is a Markovian process). The process and output noise are white and their covariance matrices are given by: $E\left[w(i) w^{T}(j)\right]=Q \delta(i-j)$ and $E\left[v(i) v^{T}(j)\right]=R \delta(i-j)$ (variable $\delta$ denotes a Dirac delta function. By using the delta function to describe the elements of the process and measurement noise covariance matrix it is stated that these matrices are diagonal ones).

Using the above, the discrete-time Kalman filter can be decomposed into two parts: (i) time update (prediction stage), and (ii) measurement update (correction stage). The first part employs an estimate of the state vector $x(k)$ made before the output measurement $z(k)$ is available (a priori estimate). The second part estimates $x(k)$ after $z(k)$ has become available (a posteriori estimate).

- When the set of measurements $Z^{-}=\{z(1), \ldots, z(k-$ $1)\}$ is available. From $Z^{-}$an a priori estimation of $x(k)$ 
is obtained which is denoted by $\hat{x}^{-}(k)=$ the estimate of $x(k)$ given $Z^{-}$.

- When $z(k)$ is available, the output measurements set becomes $Z=\{z(1), \ldots, z(k)\}$, where $\hat{x}(k)=$ the estimate of $x(k)$ given $Z$.

The associated estimation errors are defined by $e^{-}(k)=$ $x(k)-\hat{x}^{-}(k)$ is the a priori error, and $e(k)=x(k)-\hat{x}(k)$ is the a posteriori error. The estimation error covariance matrices associated with $\hat{x}(k)$ and $\hat{x}(k)$ are defined as $P^{-}(k)=$ $\operatorname{Cov}\left[e^{-}(k)\right]=E\left[e^{-}(k) e^{-}(k)^{T}\right]$ and $P(k)=\operatorname{Cov}[e(k)]=$ $E\left[e(k) e^{T}(k)\right]$ [23]. From the definition of the trace of a matrix, the mean square error of the estimates can be written as $M S E\left(\hat{x}^{-}(k)\right)=E\left[e^{-}(k) e^{-}(k)^{T}\right]=\operatorname{tr}\left(P^{-}(k)\right)$ and $\operatorname{MSE}(x(k))=E\left[e(k) e^{T}(k)\right]=\operatorname{tr}(P(k))$.

Finally, the linear Kalman filter equations are

measurement update

$$
\begin{aligned}
& K(k)=P^{-}(k) C^{T}\left[C \cdot P^{-}(k) C^{T}+R\right]^{-1} \\
& \hat{x}(k)=\hat{x}^{-}(k)+K(k)\left[z(k)-C \hat{x}^{-}(k)\right] \\
& P(k)=P^{-}(k)-K(k) C P^{-}(k)
\end{aligned}
$$

time update

$$
\begin{aligned}
& P^{-}(k+1)=A(k) P(k) A^{T}(k)+Q(k) \\
& \hat{x}^{-}(k+1)=A(k) \hat{x}(k)+B(k) u(k)
\end{aligned}
$$

\subsection{Methods for Checking the Consistency of the Kalman Filter}

To obtain accurate estimates with the Kalman filter, previously a tuning process is required. A question that arises is about which state estimates can be considered as reliable. There is need for systematic methods showing when the Kalman filter is not performing optimally and when its retuning, either in terms of the used dynamic or kinematic model or in terms of the covariance matrices, should be performed. Several methods can be applied to test the consistency of the Kalman filter, from the desired characteristics of the measurement residuals. These include the NES test, the autocorrelation test, and the normalized mean error (NME) test and have been analyzed in $[13,14]$.

(i) It is assumed that a discrete error process $e_{k}$ with dimension $m \times 1$ is a zero-mean Gaussian white-noise process with covariance given by $E_{k}$. This process can be the Kalman filter's residual associated to the state estimation error or the residual associated to the measurement estimation error. Then, the following NES is defined

$$
\epsilon_{k}=e_{k}^{T} E_{k}^{-1} e_{k}
$$

The NES follows a $\chi^{2}$ distribution. An appropriate test for the normalized error sum is to numerically show that the following condition is met within a level of confidence (according to the properties of the $\chi^{2}$ distribution)

$E\left\{\epsilon_{k}\right\}=m$

This can be succeeded using statistical hypothesis testing, which are associated with confidence intervals. A $95 \%$ confidence interval is frequently applied, which is specified using $100(1-a)$ with $a=0.05$. Actually, a two-sided probability region is considered cutting-off two end tails of $2.5 \%$ each. For $M$ runs of Monte-Carlo experiments the NES that is obtained is given by

$\bar{\epsilon}_{k}=\frac{1}{M} \sum_{i=1}^{M} \epsilon_{k}(i)=\frac{1}{M} \sum_{i=1}^{M} e_{k}^{T}(i) E_{k}^{-1}(i) e_{k}(i)$

where $\epsilon_{i}$ stands for the $i$-th run at time $t_{k}$. Then $M \bar{\epsilon}_{k}$ will follow a $\chi^{2}$ density with $M m$ degrees of freedom (DOF). This condition can be checked using a $\chi^{2}$ test. The hypothesis holds true if the following condition is satisfied

$\bar{\epsilon}_{k} \in\left[\zeta_{1}, \zeta_{2}\right]$

where $\zeta_{1}$ and $\zeta_{2}$ are derived from the tail probabilities of the $\chi^{2}$ density. For example, for $m=2$ and $M=100$ one has $\chi_{M m}^{2}(0.025)=162$ and $\chi_{M m}^{2}(0.975)=241$. Using that $M=100$ one obtains $\zeta_{1}=\chi_{M m}^{2}(0.025) / M=1.62$ and $\zeta_{2}=\chi_{M m}^{2}(0.975) / M=2.41$.

(ii) Another consistency checking method is the test for whiteness. This is obtained by using the following sample autocorrelation:

$\bar{\rho}_{k, j}=\frac{1}{\sqrt{M}} \sum_{i=1}^{M} e_{k}^{T}(i)\left[\sum_{i=1}^{M} e_{k}(i) e_{k}^{T}(i) \sum_{i=1}^{M} e_{j}(i) e_{j}^{T}(i)\right]^{-1 / 2} e_{j}(i)$

For a sufficiently large value of $M$, variable $\bar{\rho}_{j, k}$ for $k \neq j$ is zero mean with variance given by $1 / M$. Next the application of the central limit theorem provides a normal approximation, and considering a $95 \%$ confidence interval one finally obtains

$\bar{\rho}_{j, k} \in\left[-\frac{1.96}{M},+\frac{1.96}{M}\right]$

(iii) An additional consistency test is based on the normalized mean error (NME) for the $j t h$ element of $e_{k}$

$\left[\bar{\mu}_{k}\right]_{j}=\frac{1}{M} \sum_{j=1}^{M} \frac{\left[e_{k}\right]_{j}}{\sqrt{\left[E_{k}\right]_{j j}}}, \quad j=1,2, \ldots, M$

Then, since the variance of $\left[\bar{\mu}_{k}\right]_{j}$ is $\frac{1}{M}$ for a $95 \%$ acceptance interval one has 
$\left[\bar{\mu}_{k}\right]_{j} \in\left[-\frac{1.96}{\sqrt{M}}+\frac{1.96}{\sqrt{M}}\right]$

The hypothesis holds true, if Eq. (11) is satisfied. The NES, NME and autocorrelation consistency tests can be all performed with a single run using $N$ data points. Using a time-averaging approach one obtains a low variability test statistic, which can be executed in real-time. In the latter case the time-average NES is given by

$\bar{\epsilon}=\frac{1}{N} \sum_{k=1}^{N} e_{k}^{T} E_{k}^{-1} e_{k}$

Considering that $e_{k}$ is a zero mean, white-noise process, then $N \bar{\epsilon}$ follows a $\chi^{2}$ density distribution with $N m$ DOF. Through the computation of the time-average auto-correlation the whiteness test for $e_{k}$ is

$\bar{\rho}_{j}=\frac{1}{\sqrt{N}} \sum_{k=1}^{N} e_{k}^{T} e_{k+j}\left[\sum_{k=1}^{N} e_{k}^{T} e_{k} \sum_{k=1}^{N} e_{k+j}^{T} e_{k+j}\right]^{\frac{-1}{2}}$

For $N$ sufficiently large, $\bar{\rho}_{j}$ has zero mean and variance given by $1 / N$. With a $95 \%$ acceptance interval one has

$\bar{\rho}_{j} \in\left[-\frac{1.96}{\sqrt{N}}+\frac{1.96}{\sqrt{N}}\right]$

The hypothesis is accepted if Eq. (14) is satisfied. The aforementioned tests can be applied to the residuals of the Kalman filter or to the Kalman filter state errors for checking the consistency of the obtained estimation and for checking the necessary consistency for filter optimality. If the tests are not satisfied then this means that the Kalman filter is not running optimally, and the filter has to be retuned, or the filter's design has to be reconsidered.

In this paper a systematic method, the local statistical approach to fault diagnosis, will be introduced for checking the consistency of fuzzy Kalman filtering. It will be shown that the method is capable of identifying the elements responsible for the filter's failure, in the dynamic or kinematic model associated with the estimation performed by the local Kalman filters.

\section{$2.3 \chi^{2}$ Random Variables}

The $\chi^{2}$ distribution is often used to provide a consistency test in estimators. This is useful for determining if the provided state estimates are reliable and accurate or not. Assuming a Gaussian distribution for the $n \times 1$ vector $x$, with mean value $\mu$ and covariance matrix $R$, the following variable is said to have a $\chi^{2}$ distribution with $n$ DOF $[13,14]$ :

$q=(x-\mu)^{T} R^{-1}(x-\mu)$
The variable $q$ is the sum of squares of $n$ independent zeromean variables with variance equal to one. This can be shown by defining the following variable:

$u=R^{-1 / 2}(x-\mu)$

Then $u$ is a Gaussian variable with $E\{u\}=0$ and $E\left\{u u^{T}\right\}=$ $I$. The $\chi^{2}$ distribution is written as

$q \simeq \chi_{n}^{2}$

The mean and variance of variable $q$ are given by

$E\{q\}=\sum_{i=1}^{n} E\left\{u_{i}^{2}\right\}=n$

$E\left\{(q-n)^{2}\right\}=\sum_{i=1}^{n} E\left\{\left(u_{i}^{2}-1\right)^{2}\right\}=\sum_{i=1}^{n}(3-2+1)=2 n$

where the relationship $E\left\{x^{4}\right\}=3 \sigma^{4}$ has been used for the term involving $u_{i}^{4}$. This relationship is given from the scalar version of

$E\left\{x^{T} A x x^{T} B x\right\}=\operatorname{Tr}(A R) \operatorname{Tr}(B R)+2 \operatorname{Tr}(A R B R)$

where $A$ and $B$ are $n \times n$ matrices. It noted that if $A=B$ with $A=a a^{T}$, where $a$ is a $n \times 1$ vector, then Eq. (20) takes the form $E\left\{\left(a^{T} x\right)^{4}\right\}=3\left(a^{T} R a\right)^{2}$. The $\chi^{2}$ probability density function with $n$ DOF is given by

$p(q)=\frac{1}{2^{n / 2} \Gamma(n / 2)} q^{\frac{n-2}{2}} e^{-\frac{q}{2}}$

where the $\Gamma$ function is defined as

$$
\begin{gathered}
\Gamma\left(\frac{1}{2}\right)=\sqrt{\pi} \\
\Gamma(1)=1 \\
\Gamma(m+1)=m \Gamma(m)
\end{gathered}
$$

Remark 1 The need for validation of the Kalman filter against model inconsistencies becomes more apparent in its distributed implementation where decentralized information processing nodes have only local knowledge about the monitored system. By detecting local Kalman filters that perform estimation using an erroneous model it becomes possible to exclude them from the local estimates fusion procedure. Thus, the precision of the aggregate state estimate is assured, and this is particularly significant for the reliability of sensor networks and distributed autonomous navigation systems [24-27]. 


\section{Equivalence Between Kalman Filters and Regressor Models}

\subsection{Equivalence Between the Standard Kalman Filter and Linear Regressor Models}

The fuzzy Kalman filter can be transformed into a weighted sum of local ARMAX models which finally take the form of a TSK fuzzy model. The TSK model contains both moving average part and auto-regressive parts [30-37]. Actually, Kalman filters are equivalent to ARMAX models while fuzzy Kalman filters are equivalent to a sum of ARMAX models with fuzzy weighting, which finally take the form of a TSK neurofuzzy model. The description of the fuzzy Kalman filter as a TSK fuzzy model will be used in the following sections for performing the model validation tests and for checking the consistency of this distributed filter.

An autoregressive moving average model with auxiliary input (ARMAX) is an input-output model of the form [12]

$A(z) Y_{k}=C(z) U_{k}+B(z)\left\{\epsilon_{k}\right\}$

$A, B, C$ are polynomial matrices in the backwards shift operator $z^{-1}$ :

$A(z)=A_{0}-\sum_{i=1}^{p} A_{i} z^{-i}$

$B(z)=\sum_{j=0}^{q} B_{j} z^{-j}$

$C(z)=\sum_{l=1}^{l} C_{l} z^{-l}$

such that $A$ has non-singular constant term $A_{0}$ and where $\epsilon_{k}$ is a white noise sequence with covariance matrix $R$. A state-space model and particularly the Kalman filter estimator can be written in the form of an ARMAX model. For linear systems, the Kalman filter (for the single-input case) can be written in the form [12] (see: M. Basseville and I. Nikiforov, Detection of abrupt changes, Prentice Hall, 1993, Kalman filter section and Connections Between ARMA and State-Space Models section, pp. 87-93)

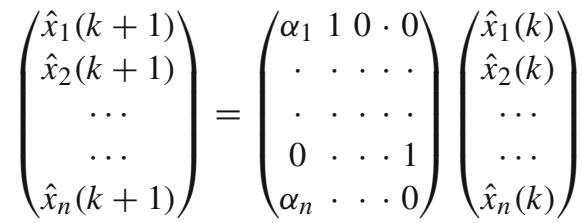

$$
\begin{aligned}
& +\left(\begin{array}{c}
g_{1} \\
\cdot \\
\cdot \\
\cdot \\
g_{n}
\end{array}\right) U_{k}+\left(\begin{array}{c}
\kappa_{1}(k) \\
\cdot \\
\cdot \\
\cdot \\
\kappa_{n}(k)
\end{array}\right) \epsilon_{k} \\
& \hat{Y}(k)=(10 \cdots 0) \hat{X}(k-1)+J U_{k}+\epsilon_{k}
\end{aligned}
$$

Using successive substitutions this can be rewritten as a timevarying ARMAX model [12]:

$$
A(z) Y_{k}=C(z) U_{k}+B(\kappa, z) \epsilon_{k}
$$

where

$$
\begin{aligned}
& A(z)=1-\alpha_{1} z^{-1}-\cdots-\alpha_{n} z^{-n} \\
& \begin{array}{c}
C(z)=g_{1} z^{-1}+\cdots+g_{n} z^{-n}+J A\left(z^{-1}\right) \\
B(\kappa, z)=1+\left[\kappa_{1}(k-1)-\alpha_{1}\right] z^{-1}+\cdots+ \\
\quad+\left[\kappa_{n}(k-n)-\alpha_{n}\right] z^{-n}
\end{array}
\end{aligned}
$$

Matrix $B(\kappa, z)$ is time-varying because the Kalman filter gain $K_{k}$ is time-varying. But, under the conditions of the stability theorem, $K$ and $B$ are asymptotically constant. Thus, the ARMAX description of the Kalman filter becomes

$A(z) Y_{k}=C(z) U_{k}+B(z) \epsilon_{k}$

This approach also holds for multi-input systems and one can transform again the state-space representation into an ARMAX model.

Generalizing to the case of fuzzy-weighted Kalman filters one has

$\hat{Y}_{k+n}=\frac{\sum_{l=1}^{L} \bar{y}^{l} \prod_{i=1}^{n} \mu_{A_{i}^{l}}\left(x_{i}\right)}{\sum_{l=1}^{L} \prod_{i=1}^{n} \mu_{A_{i}^{l}}\left(x_{i}\right)}$

where $\bar{y}^{l}(k)=c_{f_{i}}^{l} x^{T} l=1, \ldots, L$ are the outputs of the local Kalman filters weighted by fuzzy membership functions. Therefore, the fuzzy nonlinear Kalman filter becomes equivalent to a TSK fuzzy model, where the TSK model contains both a moving average part and an auto-regressive parts. Therefore each local TSK fuzzy model will correspond to fuzzy rules of the extended form

IF $Y_{k}$ is $A_{i_{1}}^{l}$ AND $Y_{k+1}$ is $A_{i_{2}}^{l} \cdots$ AND $Y_{k+n-1}$ is $A_{i_{n}}^{l}$ AND $u_{k}$ is $B_{j_{1}}^{l}$ AND $u_{k+1}$ is $B_{j_{2}}^{l} \cdots$ AND $u_{k+n-1}$ is $B_{j_{m}}^{l}$ AND $\epsilon_{k}$ is $C_{r_{1}}^{l}$ AND $\epsilon_{k+1}$ is $C_{r_{2}}^{l} \ldots$ AND $\epsilon_{k+n}$ is $C_{r_{q}}^{l}$ THEN $\hat{Y}_{k+n}$ is $A_{i_{n+1}}^{l}$

where $i_{1}, i_{2}, \ldots, i_{n}, i_{n+1} \in\{1,2, \ldots, n+1\}, j_{1}, j_{2}, \ldots$, $j_{m} \in\{1,2, \ldots, m\}$ and $r_{1}, r_{2}, \ldots, r_{q} \in\{1,2, \ldots, q\}$.

\subsection{Sensitivity of the Fuzzy Kalman Filter to Parametric Changes}

The fuzzy Kalman filter can be represented and a set of fuzzy rules of the TSK form. These are written as: 


$$
\begin{aligned}
& R_{l}: \text { IF } x_{1} \text { is } A_{1}^{l} \text { AND } x_{2} \text { is } A_{2}^{l} \text { AND } \cdots \text { AND } x_{n} \text { is } A_{n}^{l} \\
& \text { THEN } \bar{y}^{l}=\sum_{i=1}^{n} c_{f_{i}}^{l} x_{i} l=1,2, \ldots, L
\end{aligned}
$$

where $R^{l}$ is the $l$-th rule, $x=\left[x_{1}, x_{2}, \ldots, x_{n}\right]^{T}$ is the input (antecedent) variable, $\bar{y}^{l}$ is the output (consequent) variable, and $w_{i}^{l}, b^{l}$ are the parameters of the local linear models. The output of the TSK is given by the weighted average of the rules consequents [38-40]:

$\hat{y}=\frac{\sum_{l=1}^{L} \bar{y}^{l} \prod_{i=1}^{n} \mu_{A_{i}^{l}}\left(x_{i}\right)}{\sum_{l=1}^{L} \prod_{i=1}^{n} \mu_{A_{i}^{l}}\left(x_{i}\right)}$

where $\mu_{A_{i}^{l}}\left(x_{i}\right): R \rightarrow[0,1]$ is the membership function of the fuzzy set $A_{i}^{l}$ in the antecedent part of the rule $R^{l}$. The output of the $l$-th local model is given by $\bar{y}^{l}=\sum_{i=1}^{n} c_{f_{i}}^{l} x_{i}$ [41-44].

First, the residual $e_{i}$ is defined as the difference between the fuzzy model output $\hat{y}_{i}$ and the physical system output $y_{i}$, i.e. $e_{i}=\hat{y}_{i}-y_{i}$. It is also acceptable to define the residual as the difference between the fuzzy model output and the exact model output, where the exact model replaces the physical system and has the same number of parameters as the fuzzy model (see Fig. 1). The partial derivative of the residual square is:

$H\left(\theta, y_{i}\right)=\frac{1}{2} \frac{\partial e_{i}^{2}}{\partial \theta}=e_{i} \frac{\partial y_{i}}{\partial \theta}$

The vector $H$ having as elements the above $H\left(\theta, y_{i}\right)$ is called primary residual. Next, the gradients of the output with respect to the model's parameters are computed [35,45-50]. In the case of fuzzy models the gradient of the output with respect to the consequent parameters $w_{i}^{l}$ is given by

$\frac{\partial \hat{y}}{\partial w_{i}^{l}}=\frac{x_{i} \mu_{R^{l}}(x)}{\sum_{l=1}^{L} \mu_{R^{l}}(x)}$

The gradient with respect to the center $c_{i}^{l}$ is

$\frac{\partial \hat{y}}{\partial c_{i}^{l}}=\sum_{l=1}^{L} \frac{\bar{y}^{l} \frac{2\left(x_{i}-c_{i}^{l}\right)}{v_{i}^{l}} \mu_{R^{l}}\left(x_{i}\right)\left[\sum_{j=1}^{L} \mu_{R^{j}}\left(x_{i}\right)-\mu_{R^{l}}\left(x_{i}\right)\right]}{\left[\sum_{l=1}^{L} \mu_{R^{l}}\left(x_{i}\right)\right]^{2}}$

The gradient with respect to the spread $v_{i}^{l}$ is

$\frac{\partial \hat{y}}{\partial v_{i}^{l}}=\sum_{l=1}^{L} \frac{\bar{y}^{l} \frac{2\left(x_{i}-c_{c^{l}}\right)^{2}}{v_{i}^{l^{3}}} \mu_{R^{l}}\left(x_{i}\right)\left[\sum_{j=1}^{L} \mu_{R^{j}}\left(x_{i}\right)-\mu_{R^{l}}\left(x_{i}\right)\right]}{\left[\sum_{l=1}^{L} \mu_{R^{l}}\left(x_{i}\right)\right]^{2}}$
Table 1 Stages of the local statistical approach for FDI

1. Generate the residuals partial derivative given by Eq. (37)

2. Calculate the Jacobian matrix J given by Eq. (39)

3. Calculate the sensitivity matrix M given by Eq. (42)

4. Calculate the covariance matrix S given by Eq. (43)

5. Apply the $\chi^{2}$ test for change detection of Eq. (44)

6. Apply the change isolation tests of Eq. (47) or (56)

Remark 2 The equivalence between the fuzzy Kalman filter and a Takagi-Sugeno neurofuzzy model enables to exploit previous results on fault detection and isolation for nonparametric estimators, such as neurofuzzy networks, by making use the local statistical approach to fault diagnosis. By describing the fuzzy Kalman filter in the form of a Takagi-Sugeno neurofuzzy model it becomes easy to complete the intermediate stages for the application of the change detection method, which are described in Table 1 [51,52].

\section{Change Detection with the Local Statistical Approach}

\subsection{The Global $\chi^{2}$ Test for Change Detection}

The local statistical approach to fault diagnosis is a statistical method of fault diagnosis which can be used for consistency checking of the fuzzy Kalman filter. Based on a small parametric disturbance assumption, the proposed FDI method aims at transforming complex detection problems concerning a parameterized stochastic process into the problem of monitoring the mean of a Gaussian vector. The local statistical approach consists of two stages : (i) the global test which indicates the existence of a change in some parameters of the fuzzy model, (ii) the diagnostics tests (sensitivity or minmax) which isolate the parameter affected by the change. The method's stages are analyzed first, following closely the method presented in $[12,16]$.

As shown in Fig. 1 the proposed method is based on the definition of the residual $e_{i}$ described as the difference between the output from the nonlinear ARMAX model of the fuzzy Kalman filter obtained with the use of the changed dynamics or kinematics of the system and the output of the nonlinear ARMAX model of the fuzzy Kalman filter obtained with the use of the unchanged dynamics or kinematics. The nonlinear ARMAX model is actually a neuro-fuzzy model of the Takagi-Sugeno type that is based on the system's dynamics or kinematics model in an undistorted (fault-free) mode.

The concept of this FDI technique is as follows: there is a nonlinear ARMAX model that represents the unchanged system dynamics. At each time instant the output of the aforementioned reference nonlinear ARMAX model is compared 


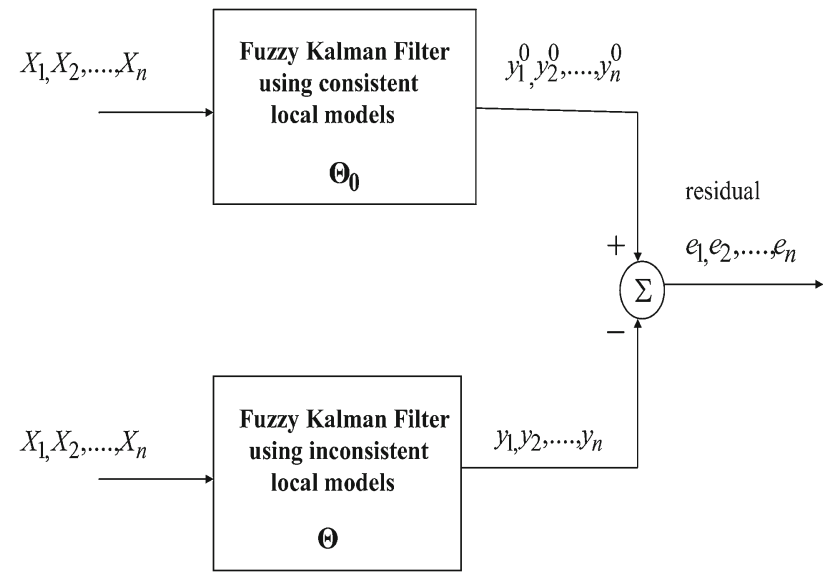

Fig. 1 Residual between the fuzzy Kalman filter that uses consistent local models and the fuzzy Kalman filter that uses inconsistent (distorted) local models

to the output of the nonlinear ARMAX model that represents the changed system dynamics. The difference between these two output measurements is called residual. The statistical processing of a sufficiently large number of residuals through a FDI method provides an index-variable that is compared against a fault threshold and which can give early indication about deviation of the model used by the Kalman filter from the real system dynamics or kinematics. Under certain conditions (detectability of changes) the proposed FDI method enables also fault isolation, i.e. to identify the source of fault within the model used by the fuzzy Kalman filter. In practical terms this means that the proposed change detection method can find out the $i$-th local Kalman filter (out of the $\mathrm{N}$ local Kalman filters that constitute the fuzzy Kalman filter) which makes use of an inconsistent model of the system's dynamics or kinematics.

Considering the representation of the fuzzy Kalman filter as a neuro-fuzzy model of the Takagi-Sugeno type, the partial derivative of the residual square is:

$H\left(\theta, y_{i}\right)=\frac{1}{2} \frac{\partial e_{i}^{2}}{\partial \theta}=e_{i} \frac{\partial \hat{y}_{i}}{\partial \theta}$

where $\theta$ is the vector of model's parameters. The vector $H$ having as elements the above $H\left(\theta, y_{i}\right)$ is called primary residual. Since the nonlinear ARMAX model is a neurofuzzy model, the gradient of the output with respect to the consequent parameters $c_{f_{i}}^{l}$ is given by

$\frac{\partial \hat{y}}{\partial c_{f_{i}}^{l}}=\frac{x_{i} \mu_{R^{l}}(x)}{\sum_{l=1}^{L} \mu_{R^{l}}(x)}$

The gradient with respect to the center $c_{i}^{l}$ has been given in Eq. (35) while the gradient with respect to the spread $v_{i}^{l}$ has been given in Eq. (36).
Next, having calculated the partial derivatives of Eqs. (38), (35) and (36), the rows of the Jacobian matrix $J$ are found by

$J\left(\theta_{0}, y_{k}\right)=\left.\frac{\partial \hat{y}_{k}(\theta)}{\partial \theta}\right|_{\theta=\theta_{0}}$

where $\theta_{0}$ represents the nominal value of the parameters. The problem of change detection with the $\chi^{2}$ test consists of monitoring a change in the mean of the Gaussian variable which for the one-dimensional parameter vector $\theta$ is formulated as

$X=\frac{1}{\sqrt{N}} \sum_{k=1}^{N} e_{k} \frac{\partial \hat{y}_{k}}{\partial \theta} \sim N\left(\mu, \sigma^{2}\right)$

where $\hat{y}_{k}$ is the output of the neural model generated by the input pattern $x_{k}, e_{k}$ is the associated residual and $\theta$ is the vector of the model's parameters. It is noted that $X$ is the monitored parameter for the FDI test, which means that when the mean value of $X$ is 0 the system is in the fault-free condition, while when the mean value of $X$ has moved away from 0 the system is in a faulty condition. For a multivariable parameter vector $\theta$ should hold $X \sim N(M \delta \theta, S)$, where $S$ denotes the covariance matrix of $X$. In order to decide if the system (Kalman filter) is in fault-free operating conditions, given a set of data of $N$ measurements, let $\theta_{*}$ be the value of the parameters vector $\mu$ minimizing the RMSE. The notation is introduced only for the convenience of problem formulation, and its actual value does not need to be known. Then the model validation problem amounts to make a decision between the two hypotheses $[12,16]$ :

$H_{0}: \theta_{*}=\theta_{0}$

$H_{1}: \theta_{*}=\theta_{0}+\frac{1}{\sqrt{N}} \delta \theta$

where $\delta \theta \neq 0$. It is known from the central limit theorem that for a large data sample, the normalized residual given by Eq. (40) asymptotically follows a Gaussian distribution when $\mathrm{N} \rightarrow \infty[12,53]$. More specifically, the hypothesis that has to be tested is:

$H_{0}: X \sim N(0, S)$

$H_{1}: X \sim N(M \delta \theta, S)$

where $M$ is the sensitivity matrix [see Eq. (42)], $\delta \theta$ is the change in the parameters' vector and $S$ is the covariance matrix [see Eq. (43)]. The product $M \delta \theta$ denotes the new center of the monitored Gaussian variable $X$, after a change on the system's parameter $\theta$. The sensitivity matrix $M$ of $\frac{1}{\sqrt{N}} X$ is defined as the mean value of the partial derivative with respect to $\theta$ of the primary residual defined in Eq. (37), i.e. $E\left\{\frac{\partial}{\partial \theta} H\left(\theta, y_{k}\right)\right\}$ and is approximated by $[12,16]$ : 
$M\left(\theta_{0}\right) \simeq \frac{\partial}{\partial \theta} \frac{1}{N} \sum_{k=1}^{N} H\left(\theta_{0}, y_{k}\right) \simeq \frac{1}{N} J^{T} J$

The covariance matrix $S$ is defined as $E\left\{H\left(\theta, y_{k}\right) H^{T}\right.$ $\left.\left(\theta, y_{k+m}\right)\right\}, m=0, \pm 1, \ldots$ and is approximated by [53]:

$$
\begin{aligned}
S & \simeq \sum_{k=1}^{N}\left[H\left(\theta_{0}, y_{k}\right) H^{T}\left(\theta_{0}, y_{k}\right)\right] \\
& +\sum_{m=1}^{I} \frac{1}{N-m} \sum_{k=1}^{N-m}\left[H\left(\theta_{0}, y_{k}\right) H^{T}\left(\theta_{0}, y_{k+m}\right)\right. \\
& \left.+H\left(\theta_{0}, y_{k+m}\right) H^{T}\left(\theta_{0}, y_{k}\right)\right]
\end{aligned}
$$

where an acceptable value for $I$ is 3 . The decision tool is the likelihood ratio $s(X)=\ln \frac{p_{\theta_{1}(x)}}{p_{\theta_{0}(x)}}$, where $p_{\theta_{1}}(X)=$ $e^{[X-\mu(X)]^{T} S^{-1}[X-\mu(X)]}$ and $p_{\theta_{0}}(X)=e^{X^{T} S^{-1} X}$ [54]. The center of the Gaussian distribution of the changed system is denoted as $\mu(X)=M \delta \theta$ where $\delta \theta$ is the change in the parameters vector. The generalized likelihood ratio (GLR) is calculated by maximizing the likelihood ratio with respect to $\delta \theta$ [53]. This means that the most likely case of parameter change is taken into account. This gives the global $\chi^{2}$ test $t$ :

$t=X^{T} S^{-1} M\left(M^{T} S^{-1} M\right)^{-1} M^{T} S^{-1} X$

Since $X$ asymptotically follows a Gaussian distribution, the statistics defined in Eq. (44) follows a $\chi^{2}$ distribution with $n$ DOF. Mapping the change detection problem to this $\chi^{2}$ distribution enables the choice of the change threshold. Assume that the desired probability of false alarm is $\alpha$ then the change threshold $\lambda$ should be chosen from the relation $[12,16]$

$\int_{\lambda}^{\infty} \chi_{n}^{2}(s) d s=\alpha$

where $\chi_{n}^{2}(s)$ is the probability density function (p.d.f.) of a variable that follows the $\chi^{2}$ distribution with $n$ DOF.

\subsection{Statistical Fault Isolation with the Sensitivity Test}

Fault isolation is needed to identify the source of faults in the dynamic or kinematic model of the system used by the fuzzy Kalman filter. This means that the fault diagnosis method should also be able to find out (among the $N$ local Kalman filters that constitute the fuzzy Kalman filter) which is the local Kalman filter that makes use of an inconsistent model. A first approach to change isolation is to focus only on a subset of the parameters while considering that the rest of the parameters remain unchanged [53]. The parameters vector $\eta$ can be written as $\eta=[\phi, \psi]^{T}$, where $\phi$ contains those parameters to be subject to the isolation test, while $\psi$ contains those parameters to be excluded from the isolation test. $M_{\phi}$ contains the columns of the sensitivity matrix $M$ which are associated with the parameters subject to the isolation test. Similarly $M_{\psi}$ contains the columns of $M$ that are associated with the parameters to be excluded from the sensitivity test.

Assume that among the parameters $\eta$, it is only the subset $\phi$ that is suspected to have undergone a change. Thus $\eta$ is restricted to $\eta=[\phi, 0]^{T}$. The associated columns of the sensitivity matrix are given by $M_{\phi}$ and the mean of the Gaussian to be monitored is $\mu=M_{\phi} \phi$, i.e.

$\mu=M A \phi, \quad A=[0, I]^{T}$

Matrix $A$ is used to select the parameters that will be subjected to the fault isolation test. The rows of $A$ correspond to the total set of parameters while the columns of A correspond only to the parameters selected for the test. Thus the fault diagnosis $\left(\chi^{2}\right)$ test of Eq. (44) can be restated as $[12,16]$ :

$t_{\phi}=X^{T} S^{-1} M_{\phi}\left(M_{\phi}^{T} S^{-1} M_{\phi}\right)^{-1} M_{\phi}^{T} S^{-1} X$

\subsection{Statistical Fault Isolation with the Min-Max Test}

In this approach the aim is to find a statistic that will be able to detect a change on the part $\phi$ of the parameters vector $\eta$ and which will be robust to a change in the non observed part $\psi[53]$. Assume the vector partition $\eta=[\phi, \psi]^{T}$. The following notation is used:

$M^{T} S^{-1} M=\left(\begin{array}{ll}I_{\varphi \varphi} & I_{\varphi \psi} \\ I_{\psi \varphi} & I_{\psi \psi}\end{array}\right)$

$\gamma=\left(\begin{array}{c}\varphi \\ \psi\end{array}\right)^{T} \cdot\left(\begin{array}{ll}I_{\varphi \varphi} & I_{\varphi \psi} \\ I_{\psi \varphi} & I_{\psi \psi}\end{array}\right) \cdot\left(\begin{array}{c}\varphi \\ \psi\end{array}\right)$

where $S$ is the previously defined covariance matrix. The min-max test aims to minimize the non-centrality parameter $\gamma$ with respect to the parameters that are not suspected for change. The minimum of $\gamma$ with respect to $\psi$ is given for $[12,16]$ :

$\psi^{*}=\arg \min _{\psi} \gamma=\varphi^{T}\left(I_{\varphi \varphi}-I_{\varphi \psi} I_{\psi \psi}^{-1} I_{\psi \varphi}\right) \varphi$

and is found to be

$$
\begin{aligned}
\gamma^{*} & =\min _{\psi} \gamma=\varphi^{T}\left(I_{\varphi \varphi}-I_{\varphi \psi} I_{\psi \psi}^{-1} I_{\psi \varphi}\right) \varphi \\
& =\left(\begin{array}{c}
\varphi \\
-I_{\psi \psi}^{-1} I_{\psi \varphi} \varphi
\end{array}\right)^{T}\left(\begin{array}{cc}
I_{\varphi \varphi} & I_{\varphi \psi} \\
I_{\psi \varphi} & I_{\psi \psi}
\end{array}\right)\left(\begin{array}{c}
\varphi \\
-I_{\psi \psi}^{-1} I_{\psi \varphi} \varphi
\end{array}\right)
\end{aligned}
$$


which results in

$$
\begin{aligned}
\gamma^{*}= & \varphi^{T}\left\{\left[I,-I_{\varphi \psi} I_{\psi \psi}^{-1}\right] M^{T} \Sigma^{-1}\right\} \Sigma^{-1} \\
& \left\{\Sigma^{-1} M\left[I,-I_{\varphi \psi} I_{\psi \psi}^{-1}\right]\right\} \varphi
\end{aligned}
$$

The following linear transformation of the observations is considered:

$X_{\phi}^{*}=\left[I,-I_{\varphi \psi} I_{\psi \psi}^{-1}\right] M^{T} \Sigma^{-1} X$

The transformed variable $X_{\phi}^{*}$ follows a Gaussian distribution $N\left(\mu_{\phi}^{*}, I_{\phi}^{*}\right)$ with mean:

$\mu_{\varphi}^{*}=I_{\varphi}^{*} \varphi$

and with covariance:

$I_{\varphi}^{*}=I_{\varphi \varphi}-I_{\varphi \psi} I_{\psi \psi}^{-1} I_{\psi \varphi}$

The max-min test decides between the hypotheses:

$H_{0}^{*}: \mu^{*}=0$

$H_{1}^{*}: \mu^{*}=I_{\varphi}^{*} \varphi$

and is described by:

$\tau_{\varphi}^{*}=X_{\varphi}^{* T} I_{\varphi}^{*-1} X_{\varphi}^{*}$

The stages of fault detection and isolation (FDI) with the use of the local statistical approach are summarized in the following table:

Remark 3 The local statistical approach to fault diagnosis stands for a complete method for fault detection and isolation which enables to find out the existence of small additive faults and parametric variations (multiplicative faults) in the monitored system. Comparing to the majority of other fault diagnosis methods in time domain, which are limited to estimation of the fault-free system dynamics for developing a reference model and to residuals generation, the local statistical approach provides also the statistical tools for analyzing the information contained in the residuals and an optimally chosen threshold for deciding efficiently about the existence of a failure. This enables to minimize false conclusions and also to detect incipient changes which could be hidden by measurement noise [55-57].

Remark 4 The local statistical approach to fault diagnosis has been developed aiming at incipient faults detection. The problem of parametric change detection with the use of the likelihood ratio as a decision making criterion becomes equivalent to the problem of monitoring the statistical properties of the multidimensional residual variables given in Eq.
(40), when small variations are assumed about the system's parameters. In such a case the Taylor series expansion of the likelihood ratio is well approximated by the residuals vector variable of Eq. (40). It is noted however, that the purpose of detecting incipient faults in the models that are in use by the fuzzy Kalman filter is to avoid critical conditions in the monitored system. The effects of small modeling errors in the recursion of the local Kalman filters can be corrected before having major threats for the system's safety.

\section{Simulation Tests}

\subsection{Fuzzy Kalman Filter for an Electric Motor Model}

The fuzzy Kalman filter will be used to track the dynamics of a nonlinear DC motor model. DC motors find several applications in automotive systems, such as electronic throttle control, wheels actuation, vehicle steering, braking and active suspension control [58-62].

In the following example nonlinear DC motor model is described by the dynamics $\dot{x}=f(x)+g(x) u$ where $f(x)$ and $g(x)$ are vector fields and $x$ is the state vector. It is assumed that by linearizing at local operating points, local linear models of the electric motor are obtained. The fuzzy Kalman filter makes use of these local models to produce a state estimate for the nonlinear electric motor. A local linear model of the electric motor's dynamics is given in [4]:

$$
\left(\begin{array}{c}
\dot{x}_{1} \\
\dot{x}_{2} \\
\dot{x}_{3}
\end{array}\right)=\left(\begin{array}{ccc}
0 & 1 & 0 \\
0 & 0 & 1 \\
0 & \frac{-k_{e}^{2}-k_{d} R}{J L} & \frac{-J R-k_{d} L}{J L}
\end{array}\right)\left(\begin{array}{c}
x_{1} \\
x_{2} \\
x_{3}
\end{array}\right)+\left(\begin{array}{c}
0 \\
0 \\
\frac{k_{e}}{J L}
\end{array}\right) V
$$

The state vector as $\left[x_{1}, x_{2}, x_{3}\right]^{T}=[\theta, \dot{\theta}, \ddot{\theta}]^{T}$ while the parameters of the model are defined as: $L$ is the armature inductance, $I$ is the armature current, $k_{e}$ is the motor electrical constant, $k_{d}$ is the motor's mechanical constant, $R$ is the armature resistance, $V$ is the input voltage, taken as control input, $J$ is the motor inertia, $\omega$ is the rotor rotation speed, and $k_{d}$ is the mechanical dumping constant.

Denoting $\alpha_{1}=-\frac{k_{e}^{2}-k_{d} R}{J L}, \alpha_{2}=-\frac{J R-k_{d} L}{J L}$ and $b_{1}=\frac{k_{e}}{J L}$, a discrete-time linearized model of the motor is obtained after applying discretization with the 1-st order Euler method

$$
\begin{aligned}
\left(\begin{array}{c}
x_{1}(k+1) \\
x_{2}(k+1) \\
x_{3}(k+1)
\end{array}\right)= & \left(\begin{array}{ccc}
1 & T_{s} & 0 \\
0 & 1 & T_{s} \\
0 & T_{s} \alpha_{1} & 1+T_{s} \alpha_{2}
\end{array}\right) \\
& \cdot\left(\begin{array}{l}
x_{1}(k) \\
x_{2}(k) \\
x_{3}(k)
\end{array}\right)+\left(\begin{array}{c}
0 \\
0 \\
T_{s} b_{1}
\end{array}\right) u(k)
\end{aligned}
$$




$$
y(k)=\left(\begin{array}{lll}
1 & 0 & 0
\end{array}\right) \cdot\left(\begin{array}{l}
x_{1}(k) \\
x_{2}(k) \\
x_{3}(k)
\end{array}\right)
$$

Each linearized discrete-time model of the DC motor is associated with a different operating point and takes also into account variations in the mechanical parameters of the motor or in parameters of the motor's circuit. Thus, one can consider that the motor's dynamics is described by $N$ local linear models $i=1,2, \ldots, N$.

$x^{i}(k+1)=A^{i}(k) x^{i}(k)+B^{i}(k) u(k)$

$y^{i}(k)=C^{i}(k) x^{i}(k)$

Using the previous state-space description with the use of local linear models, ARMA models of the motor's dynamics can be obtained. Next, the Kalman filter that tracks the motor's dynamics is considered. The associated local ARMAX model is:

$y(k+3)=\left(3+T_{s} \alpha_{2}\right) y(k+2)-\left(3+3 T_{s}-\alpha_{2}-T_{s}^{2} \alpha_{1}\right) y(k+$ $1)+\left(1+T_{s} \alpha_{2}-T_{s}^{2} \alpha_{1}\right) y(k)+T_{s}^{3} b_{1} u(k)+\kappa_{1}(k) \hat{e}(k+$ $2)+\left[\left(-2-T_{s} \alpha_{2}\right) \kappa_{1}(k)-T_{s} \kappa_{2}(k)\right] \hat{e}(k+1)+\left[\left(T_{s} \alpha_{2}-\right.\right.$ $\left.\left.T_{s}^{2} \alpha_{1}\right) \kappa_{1}(k)+\left(T_{s}+T_{s}^{2} \alpha_{1}\right) \kappa_{2}(k)+T_{s}^{2} \kappa_{3}(k)\right] \hat{e}(k)$

Denoting $c_{f}^{1}=3+T_{s} \alpha_{2}, c_{f}^{2}=-\left(3+3 T_{s}-\alpha_{2}-T_{s}^{2} \alpha_{1}\right), c_{f}^{3}=$ $1+T_{s} \alpha_{2}-T_{s}^{2} \alpha_{1}, c_{f}^{4}=T_{s}^{3} b_{1}, c_{f}^{5}=\kappa_{1}(k), c_{f}^{6}=[(-2-$ $\left.\left.T_{s} \alpha_{2}\right) \kappa_{1}(k)-T_{s} \kappa_{2}(k)\right]$ and $c_{f}^{7}=\left[\left(T_{s} \alpha_{2}-T_{s}^{2} \alpha_{1}\right) \kappa_{1}(k)-\right.$ $\left.\left(T_{s}+T_{s}^{2} \alpha_{1}\right) \kappa_{2}(k)+T_{s}^{2} \kappa_{3}(k)\right]$, the description of the $l$-th local ARMAX model of the DC motor becomes

$$
\begin{aligned}
y^{l}(k+3)= & c_{f_{1}}^{l} y(k+2)+c_{f_{2}}^{l} y(k+1)+c_{f_{3}}^{l} y(k)+c_{f_{4}}^{l} u(k) \\
& +c_{f_{5}}^{l} \hat{e}(k+2)+c_{f_{6}}^{l} \hat{e}(k+1)+c_{f_{7}}^{l} \hat{e}(k)
\end{aligned}
$$

Assuming $n=7$ input variables, each one partitioned into 3 fuzzy sets and following the input dimension partitioning the input space will be partitioned into $N=3^{7}=2187$ fuzzy regions, which also means $N=2187$ local models. To avoid the explosion of dimensionality due to input dimension partitioning one can assume a limited number of local models $M<<N$ according to the concept of input space partitioning.

Thus, one obtains the following nonlinear ARMAX model for the fuzzy Kalman filter

$y(k+3)=\frac{\sum_{l=1}^{M} c_{f}^{l} \underline{x}^{T} \prod_{i=1}^{n} \mu_{A_{i}}^{l}(\underline{x})}{\sum_{l=1}^{M} \prod_{i=1}^{n} \mu_{A_{i}}^{l}(\underline{x})}$

where $c_{f}^{l}$ and $\underline{x}$ are vectors defined as

$\underline{c_{f}^{l}}=\left(c_{f_{1}}^{l} c_{f_{2}}^{l} c_{f_{3}}^{l} c_{f_{4}}^{l} c_{f_{5}}^{l} c_{f_{6}}^{l} c_{f_{7}}^{l}\right)$ $\underline{x}=(y(k+2) y(k+1) y(k) u(k) \hat{e}(k+2) \hat{e}(k+1) \hat{e}(k))$

Residuals are generated as differences between the output of the fuzzy Kalman filter associated with the undistorted dynamics of the system and fuzzy Kalman filter associated with the changed dynamics of the system.

\subsection{Validation Tests for the Fuzzy Kalman Filter of the Electric Motor}

The fuzzy Kalman filter associated with the description of the DC motor dynamics through local linear models was taken to consist of the following rules:

$$
\begin{aligned}
& R^{(1)}: \operatorname{IF} x_{1} i s\left(c_{1}^{(1)}, v\right) \text { AND } x_{2} \text { is }\left(c_{2}^{(1)}, v\right) \text { AND } \cdots \\
& \text { AND } x_{n} i s\left(c_{n}^{(1)}, v\right) \cdots \text { THEN } \hat{y}=\underline{c_{f}^{(1)}} \underline{x^{T}} \\
& R^{(2)}: \operatorname{IF} x_{1} i s\left(c_{1}^{(2)}, v\right) \text { AND } x_{2} i s\left(c_{2}^{(2)}, v\right) \text { AND } \cdots \\
& \text { AND } x_{n} i s\left(c_{n}^{(2)}, v\right) \cdots \text { THEN } \hat{y}=\underline{c_{f}^{(2)}} \underline{x^{T}} \\
& R^{(3)}: \operatorname{IF} x_{1} i s\left(c_{1}^{(3)}, v\right) \text { AND } x_{2} i s\left(c_{2}^{(3)}, v\right) \text { AND } \cdots \\
& \text { AND } x_{n} i s\left(c_{n}^{(3)}, v\right) \cdots \text { THEN } \hat{y}=\underline{c_{f}^{(3)}} \underline{x^{T}} \\
& R^{(4)}: \operatorname{IF} x_{1} i s\left(c_{1}^{(4)}, v\right) \text { AND } x_{2} i s\left(c_{2}^{(4)}, v\right) \text { AND } \cdots \\
& \text { AND } x_{n} \text { is }\left(c_{n}^{(4)}, v\right) \cdots \text { THEN } \hat{y}=\underline{c_{f}^{(4)}} \underline{x^{T}}
\end{aligned}
$$

The above model implies a local linearization of the initial nonlinear function using 4 sub-models. The spread of the membership functions is denoted by $v$. A 2D projection of the input space partition is demonstrated in Fig. 2.

The size of the model was limited to 4 rules. To further reduce the number of parameters in the fault diagnosis test, in the antecedent part of the rules constituting the fuzzy Kalman filter only the first three variables were maintained i.e. $y(k+2), y(k+1)$ and $y(k)$. Thus, the parameters set in the new TSK fuzzy model consisted of $4 \times 7+4 \times 3=40$ parameters (28 linear parameters which were the output layer weights and 12 nonlinear parameters which were the centers of the fuzzy sets in the antecedent part of the rules). This means that by applying the local statistical approach to FDI and the $\chi^{2}$ change detection test to the considered model, the fault threshold should be equal to 40 .

The numerical tests confirmed theory. In case that no fault was assumed for the monitored system the mean value of the $\chi^{2}$ test over a number of trials was found to be close to the threshold value 40 . Such a value was anticipated according to the theoretical analysis of the $\chi^{2}$ test. For slight deviations of the parameters of the fuzzy Kalman filter from their 


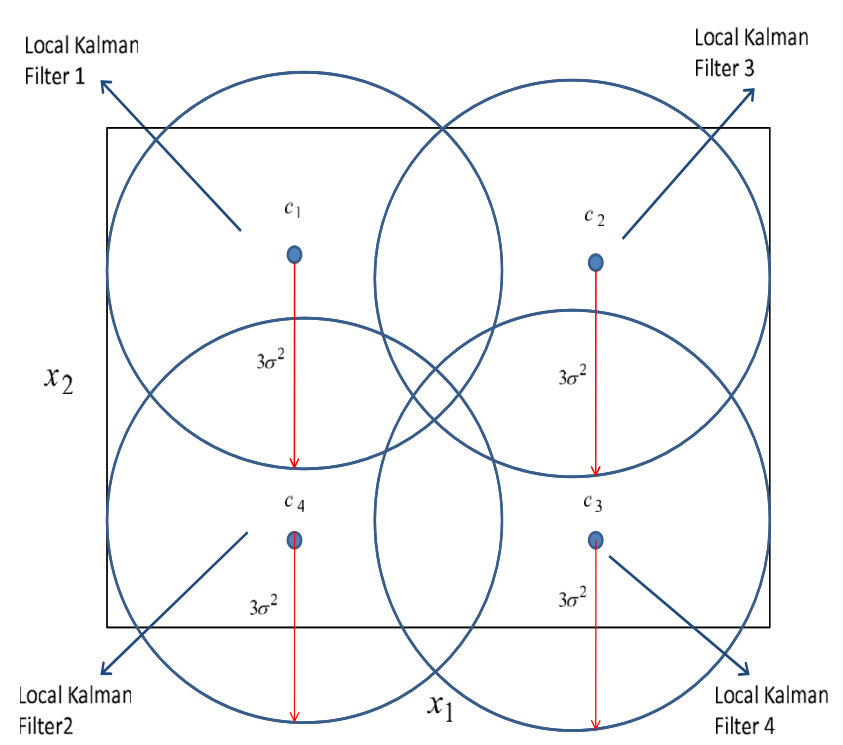

Fig. 2 Fuzzy rule base generated with input space partition

Table 2 Comparison between $\chi^{2}$ and MSE tests

\begin{tabular}{lrl}
\hline$\%$ change & \multicolumn{1}{l}{$\chi^{2}$} & \multicolumn{1}{l}{ MSE } \\
\hline 0.20 & 44.45 & $1.01 \times 10^{-6}$ \\
0.24 & 60.54 & $1.01 \times 10^{-6}$ \\
0.30 & 101.21 & $1.01 \times 10^{-6}$ \\
0.35 & 106.99 & $1.01 \times 10^{-6}$ \\
0.40 & 172.49 & $1.01 \times 10^{-6}$ \\
0.45 & 165.97 & $1.02 \times 10^{-6}$ \\
0.50 & 187.87 & $1.02 \times 10^{-6}$ \\
0.55 & 230.14 & $1.02 \times 10^{-6}$ \\
0.60 & 295.33 & $1.02 \times 10^{-6}$ \\
0.65 & 282.00 & $1.03 \times 10^{-6}$ \\
0.70 & 330.59 & $1.03 \times 10^{-6}$ \\
0.75 & 365.88 & $1.03 \times 10^{-6}$ \\
\hline
\end{tabular}

nominal (fault-free) values, the global $\chi^{2}$ test was capable of giving a clear indication about the existence of a fault. Thus for changes which varied between 0.1 and $1 \%$ of the nominal parameter's value the score of the $\chi^{2}$ test deviated significantly from the fault threshold (which as mentioned before was set equal to 40).

A comparison between (i) the proposed $\chi^{2}$ test based on the local statistical approach and the Generalized Likelihood ratio and (ii) of the mean square error (MSE) test, for detecting model inconsistencies in the distributed / fuzzy Kalman filter is given in Table 2 and in Fig. 3. It can be clearly noticed that for small parametric changes in the local models used by the fuzzy Kalman filter, the MSE test gives the erroneous conclusion that the functioning of the Kalman filter remains accurate. Actually it is observed that there is no change in the
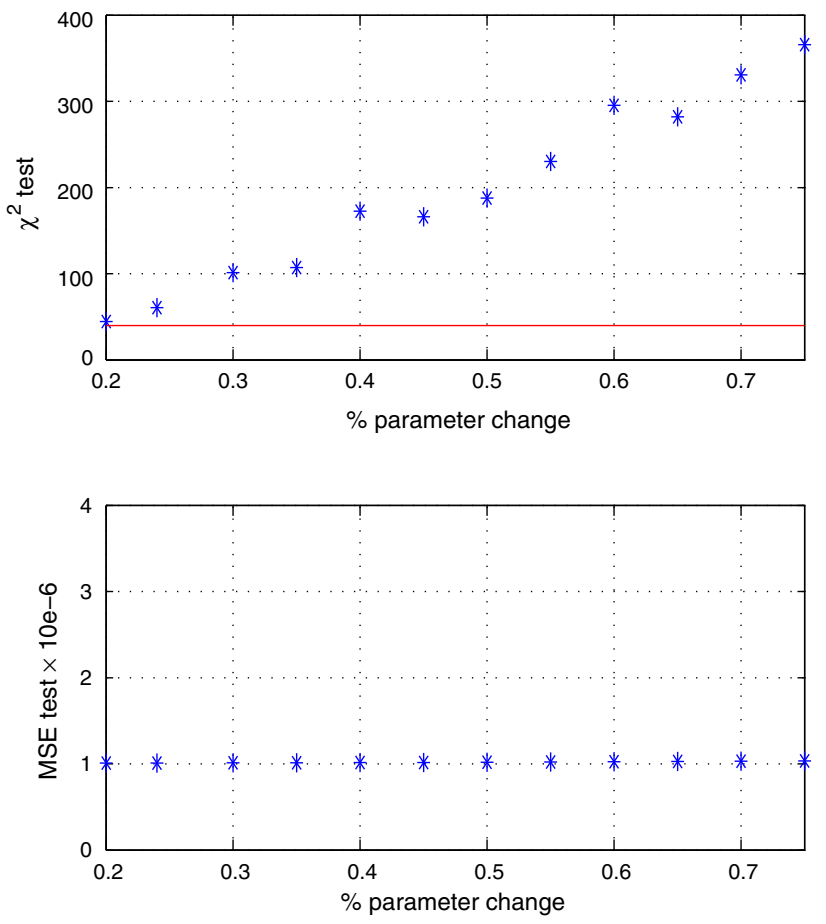

Fig. 3 Comparison between (i) the proposed $\chi^{2}$ test based on the local statistical approach and the Generalized Likelihood ratio and (ii) of the mean square error (MSE) test, for detecting model inconsistencies in the distributed / Fuzzy Kalman filter

MSE value despite changes in the parameters of the model used by the Kalman filter, and the MSE value remains low as in the case of fault-free operation. Besides in the MSE test the fault threshold is defined in an ad-hoc manner and this is another reason for the low credibility of this test. On the other hand the proposed $\chi^{2}$ test based on the local statistical approach and the Generalized Likelihood ratio provides a clear indication about inconsistencies between the models used by the fuzzy Kalman filter and the dynamics of the real system. Despite the small magnitude of parametric changes, the output of the $\chi^{2}$ test based on the local statistical approach becomes several times larger than the fault threshold (that is 40). Thus a clear indication is provided about the need to correct the parameters of the local models used by the Fuzzy Kalman filter.

As far as fault isolation is concerned, the numerical results showed that the sensitivity method for fault isolation was very efficient in distinguishing the parameter subject to fault among all parameters in the fuzzy Kalman filter's model. The sensitivity fault isolation test and the min-max fault isolation test was performed for the parameters (weight $w_{i}$ ) of the local Kalman filter. As it can be observed from the test's success rate depicted in Fig. 4 the proposed fault isolation methods can detect the local Kalman filter, that uses an inconsistent model with reference to the real system's model. Thus correction of the parameters of this particular filter can be carried 


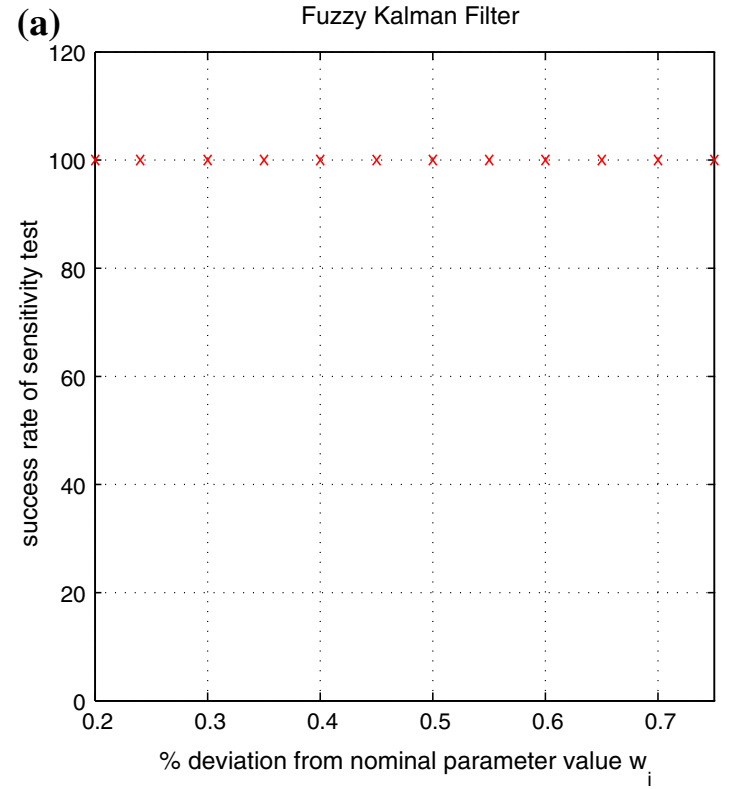

Fig. 4 a Success rate of the fault isolation test (sensitivity method) for changes in a parameter of the first local Kalman filter, ranging between 0.1 and $1.0 \%$ of the nominal value. b Success rate of the fault isola-

out instead of redesign of all local Kalman filters constituting the fuzzy Kalman filter.

Remark 5 The proposed model validation method for the fuzzy Kalman filter is based on the GLR, which means the likelihood ratio for the normalized residuals vector $X$ of Eq. (40) maximized with respect to the model's parameter vector. This is shown finally to arrive at a $\chi^{2}$ test of the form of Eq. (44). The isolation of faulty parameters in the local Kalman filters is also based on $\chi^{2}$ tests, applied to subspaces in the system's state-space description. The presented evaluation tests of the local statistical approach for model validation given in Table 2 and in Fig. 3 show that the method is capable of detecting parametric model discrepancies which are less than $1 \%$ of the associated nominal values. The local statistical approach to fault diagnosis exhibits two advantages: (i) it provides a credible criterion ( $\chi^{2}$ test) which is more efficient than other methods for Kalman filter Validation (such as the normalized error square (NES) test, the autocorrelation test or the normalized mean error (NME) test )since it employs the modeling error derivative and records the tendency for change. Thus early change detection for the filter's parameters becomes possible, (ii) It enables fault isolation since it recognizes the parameters of the fuzzy Kalman filter that are responsible for the deviation of the filter's estimates from the real output of the monitored dynamical system. Thus the retuning or redesign of the fuzzy Kalman filter can focus on a small number of its parameters.

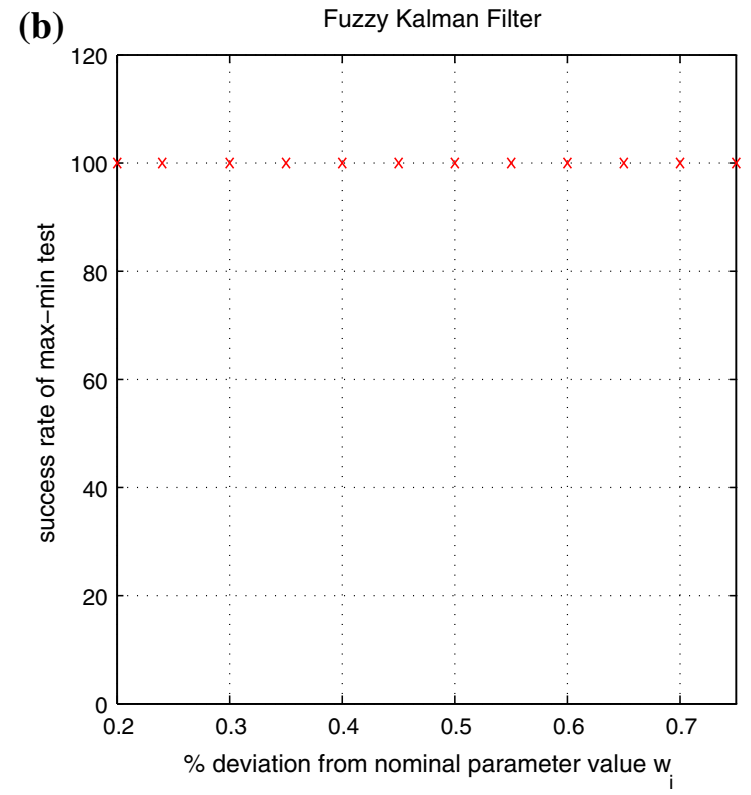

tion test ( $\max -\min$ method) for changes in a parameter of the first local Kalman filter, ranging between 0.1 and $1.0 \%$ of the nominal value

\section{Conclusion}

The objective of this research work was to develop an approach for validating the accuracy of distributed modelbased nonlinear state estimators, such as the fuzzy Kalman filter. It has been shown that the fuzzy Kalman filter is designed based on the coverage of the state space by several local linear estimators according to the concept of fuzzy local linearization. Actually, the fuzzy Kalman filter consists of local linear Kalman estimators which are weighted by fuzzy membership functions. It has been demonstrated that the fuzzy Kalman filter can be written in the form of a TSK fuzzy model, which stands for local ARMAX models weighted by fuzzy membership functions.

The paper has proposed the local statistical approach to fault diagnosis for accuracy checking of the fuzzy Kalman filter. Validation of the fuzzy Kalman filter with the Local Statistical Approach has two significant advantages: (i) it provides a credible criterion (upgraded $\chi^{2}$ test) to detect if the fuzzy Kalman filter is reliable or not. This criterion is more efficient than the aforementioned normalized square error and mean error tests since it employs the modeling error derivative and records the tendency for change. Thus early change detection for the filter's parameters becomes possible (ii) it recognizes the parameters of the fuzzy Kalman filter that are responsible for the deviation of the filter's estimates from the real output of the monitored dynamical system. Thus the retuning or redesign of the fuzzy Kalman filter can focus on a small number of its parameters. 
The efficiency of the proposed method for validation of the fuzzy Kalman filter has been tested, through simulation experiments, in the problem of tracking the dynamics of a nonlinear DC electric motor model. The proposed method was capable of not only detecting inaccuracy in the aggregate estimation provided by the fuzzy Kalman filter, but could also find out the local Kalman filter (among N distributed Kalman filters) that was using an inconsistent model of the monitored system. Application domains for which this validation procedure would be particularly useful are sensor networks, autonomous navigation systems, precision mechatronics and decentralized monitoring of industrial production.

\section{References}

1. Harris, C.J., Gan, Q.: State estimation and multi-sensor data fusion using data-based neurofuzzy local linearization process models. Inf. Fusion 2, 17-29 (2001)

2. Watanabe, K., Pathiranage, C.D., Izumi, K.: A fuzzy Kalman filter approach to the SLAM problem of nonholonomic mobile robots. In: Proceedings of the 17th IFAC World Congress, pp. 4600-4605, Seoul, July 2008

3. McGinnity, S., Irwin, G.: Nonlinear Kalman filtering using fuzzy local linear models. In: Proceedings of the American Control Conference, pp. 3299-3300, Albuquerque, June 1997

4. Rigatos, G.G.: Modelling and Control for Intelligent Industrial Systems: Adaptive Algorithms in Robotics and industrial engineering. Springer, Heidelberg (2011)

5. Zolghadri, A.: An algorithm for real-time failure detection in Kalman filters. IEEE Trans. Autom. Control 41(10), 1537-1539 (1996)

6. Brumback, B.D., Srinath, M.D.: A Chi-square test for faultdetection in Kalman filters. IEEE Trans. Autom. Control 32(6), 552-554 (1987)

7. Jwo, D.J., Cho, T.S.: A practical note on evaluating Kalman filter performance optimality and degradation. Appl. Math. Comput. 193(2), 482-505 (2007)

8. Liu, J., Li, D., Xiong, Z.: Research on an improved residual Chisquare fault detection method for federated unscented Kalman filter. Chin. J. Sci. Instrum. 30(12), 2568-2573 (2009)

9. Zhuang, C., Fu, L., Fan, Y.: Multiple fading Kalman filter based on hypothesis testing. J. Beijing Univ. Aeronaut. Astronaut. 30(1), 18-22 (2004)

10. Valestrand, R., Naevdal, G., Stordal, A.S.: Evaluation of EnKF and variants on the PUNQ-S3 case. Oil Gas Sci. Technol. Rev. IFP Energ. nouv. 67(5), 841-855 (2012)

11. Gibbs, R.G.: New Kalman filter and smoother consistency tests. Automatica 49(10), 3141-3144 (2013)

12. Basseville, M., Nikiforov, I.: Detection of Abrupt Changes. Prentice Hall, Upper Saddle River (1993)

13. Bar-Shalom, Y., Rong Li, X., Kirubarajan, T.: Estimation with Applications to Tracking and Navigation. Wiley, New York (2001)

14. Crassidis, J.L., Junkins, J.L.: Optimal Estimation of Dynamic Systems, 2nd edn. CRC Press, Boca Raton (2012)

15. Benveniste, A., Basseville, M., Moustakides, G.: The asymptotic local approach to change detection and model validation. IEEE Trans. Autom. Control 32(7), 583-592 (1987)

16. Zhang, Q., Basseville, M., Benveniste, A.: Fault detection and isolation in nonlinear dynamic systems : a combined input-output and local approach. Automatica 34(11), 1359-1373 (1998)
17. Rigatos, G., Zhang, Q.: Fuzzy Model Validation Using the Local Statistical Approach, Publication Interne IRISA No 1417. Rennes, France (2001)

18. Rigatos, G., Zhang, Q.: Fuzzy model validation using the local statistical approach. Fuzzy Sets Syst. 60(7), 882-904 (2009)

19. Rigatos, G.G., Siano, P., Piccolo, A.: A neural network-based approach for early detection of cascading events in electric power systems. IET J. Gener. Transm. Distrib. 3(7), 650-665 (2009)

20. Rigatos, G.: A Kalman filtering approach of improved precision for fault diagnosis in distributed parameter systems. Internal Report, Industrial Systems Institute (2013) arXiv:1310.3358

21. Rigatos, G.G., Tzafestas, S.G.: Extended Kalman filtering for fuzzy modelling and multi-sensor fusion. Math. Comput. Model. Dyn. Syst. 13, 251-266 (2007)

22. Rigatos, G.G.: Extended Kalman and particle filtering for sensor fusion in motion control of mobile robots. Math. Comput. Simul. 81(3), 590-607 (2010)

23. Kamen, E.W., Su, J.K.: Introduction to Optimal Estimation. Springer, London (1999)

24. Mahler, R.P.S.: Statistical Multisource-Multitarget Information Fusion. Artech House Inc, Boston (2007)

25. Makarenko, A., Durrany-Whyte, H.: Decentralized Bayesian algorithms for active sensor networks. Inf. Fusion 7(4), 418-433 (2006)

26. Gao, S., Zhong, Y., Zhang, X., Shirinzadeh, B.: Multi-sensor optimal data fusion for INS/GPS/SAR integrated navigation system. Aerosp. Sci. Technol. 13(4-5), 232-237 (2009)

27. Pathirana, P.N., Savkin, A.V., Ekanayake, S.W., Bauer, N.J.: A robust solution to the stereo-vision-based simultaneous localization and mapping problem with steady and moving landmarks. Adv. Robot. 25(6-7), 765-788 (2011)

28. Rodger, J.A.: Toward reducing failure risk in an integrated vehicle health maintenance system: a fuzzy multi-sensor data fusion Kalman filter approach for IVHMS. Expert Syst. Appl. 39(10), 98219836 (2012)

29. Schuurman, D.C., Capson, D.W.: Robust direct visual servo using network-synchronized cameras. IEEE Trans. Rob. Autom. 20(2), 319-334 (2004)

30. Harris, C.J., Hang, X., Gon, X.: Adaptive Modelling, Estimation and Fusion from Data: A Neurofuzzy Approach. Springer, Berlin (2002)

31. Zhang, Q.: Nonlinear system identification with an integrable continuous time nonlinear ARX model. J. Eur. Syst. Autom. 46(6-7), 691-710 (2012)

32. Haykin, S.: Neural Networks: A Comprehensive Foundation. McMillan, New York (1994)

33. Wang, L.X.: A Course in Fuzzy Systems and Control. PrenticeHall, New York (1998)

34. Takagi, T., Sugeno, M.: Fuzzy identification of systems and its applications to modeling and control. IEEE Trans. Syst. Man Cybern. 15, 116-132 (1985)

35. Chen, M.Y., Linkens, D.A.: A systematic neuro-fuzzy modeling framework with application to material property prediction. IEEE Trans. Syst. Man Cybern. Part B 31(5), 781-790 (2001)

36. Johansen, T.A.: Fuzzy model based control: stability. Robustness, and performance issues. IEEE Trans. Fuzzy Syst. 2(3), 221-234 (1994)

37. Chak, C.K., Feng, G., Mia, J.: An adaptive fuzzy neural network for MIMO system model approximation in high dimensional spaces. IEEE Trans. Syst. Man Cybern. Part B 28(3), 436-446 (1998)

38. Glorennec, P.Y.: Algorithmes d' apprentissage pour systèmes d' inférence floue. Hermes Science Publications, Paris (1999)

39. Nelles, O., Fischer, M.: Local linear model trees (LOLIMOT) for linear system identification of a cooling blast', In: Proceedings of the EUFIT 96 Conference, Aachen (1996) 
40. Isermann, R., Schreiber, A.: Identification methods for experimental modelling of nonlinear combustion processes. In: Proceedings of the Collection of Technical Papers - 4th International Energy Conversion. Engineering Conference, vol. 2, pp. 1468-1485 (2006)

41. Jang, J.-S.R., Sun, C.T., Mizutani, E.: Neurofuzzy and SoftComputing, A Computational Approach to Learning and Machine Intelligence. Prentice-Hall, Upper Saddle River (1997)

42. Setnes, M., Babuska, R.: Fuzzy modeling for predictive control. In: Farinwata, Filev, Langari, (eds.) Fuzzy Control—Synthesis and Analysis, pp. 23-46. Wiley, Chichester (2000)

43. Setnes, M., Kaymak, U.: Extended fuzzy c-means with volume prototypes and cluster merging. In: Proceedings of the EUFIT'98, Aachen (1998)

44. Isermann, R.: Local basis function networks for identification of a turbocharger'. In: Proceedings of the IEE Conference, vol. 427, (1), pp. 7-12 (1996)

45. Bertsekas, D.P.: Incremental least squares methods and the extended Kalman filter. SIAM J. Optim. 6(3), 807-822 (1996)

46. Simon, D.: Training fuzzy systems with the extended Kalman filter. Fuzzy Sets Syst. 132, 189-199 (2002)

47. Dubois, D., Prade, H., Ughetto, L.: Checking the coherence and redundancy of fuzzy knowledge bases. IEEE Trans. Fuzzy Syst. 5(3), 398-417 (1997)

48. Setnes, M., Babuska, R., Kaymak, U., van Nauta Lemke, H.R.: Similarity measures in fuzzy rule base simplification. IEEE Trans. Syst. Man Cybern. Part B 28(3), 376-386 (1998)

49. Psichogios, D.C., Ungar, L.H.: SVD-NET: an algorithm that automatically selects network structure. IEEE Trans. Neural Netw. 5(3), 513-515 (1994)

50. Yen, J., Wang, L.: Simplifying fuzzy rule-based models using orthogonal transformation methods. IEEE Trans. Syst. Man Cybern. Part B 29, 13-24 (1999)

51. Basseville, M.: On fault detectability and isolability. Eur. J. Control 7(6), 625-637 (2001)

52. Basseville, M., Benveniste, A., Goursat, M., Mevel, L.: In-flight vibration monitoring of aeronautical structures. IEEE Control Syst. Mag. 27(5), 27-42 (2007)
53. Basseville, M., Benveniste, A., Zhang, Q.: Surveilliance d' installations industrielles : démarche générale et conception de l' algorithmique. IRISA Publication Interne No 1010 (1996)

54. Zhang, Q., Basseville, M.: Statistical fault detection and isolation for linear time-varying systems, In: Proceedings of the 16th IFAC Conference on Systems Identification, Brussels, July 2012

55. Zhang, Q., Basseville, M.: Advanced numerical computation of chi2-tests for fault detection and isolation. In: SAFEPROCESS'03 - 5th IFAC/IMACS Symposium on Fault Detection, Supervision and Safety of Technical Processes, Washington DC (2003)

56. Basseville, M., Benveniste, A., Zhang, Q.: Towards the handling of uncertainties in statistical FDI, In: 18th IFAC World Congress Milano (2011)

57. Van der Auweraer, H., Hermans, L., Mevel, L., Benveniste, A., Basseville, M.: A model based approach to structural damage detection and localization. In: 13th International Congress and Exhibition on Condition Monitoring and Diagnostic Engineering ManagementCOMADEM'00. Houston (2001)

58. Zhang, G., Gao, J., Yu, F.: Design of active and energy-regenerative controllers for DC-motor-based suspension. Mechatronics 22, 1124-1134 (2012)

59. Sok, J.B., Seok Byun, K.S.: Throttle actuator control system for vehicle traction control. Mechatronics 9, 477-495 (1999)

60. Pavkovic, D., Deur, J., Jansz, M., Peric, N.: Adaptive control of automotive electronic throttle. Control Eng. Pract. 14, 121-136 (2006)

61. Wu, F.K., Yeh, T.J., Huang, C.F.: Motor control and torque coordination of an electric vehicle actuated by two in-wheel motors. Mechatronics 23(1), 4660 (2013)

62. Zeraoulia, M., El Hachemi, M., Benbouzid, Diallo, D.: Electric motor drive selection issues for HEV propulsion systems: a comparative study. IEEE Trans. Veh. Technol. 55(6), 1756-1764 (2006) 